\title{
Microarray analysis to evaluate different animal models for human heart failure
}

The inability of either or both sides of the heart to pump sufficient blood for maintenance of body functions is considered as heart failure (HF). Mutations in several genes have been associated with HF. Other prominent causes are hypertension, coronary atherosclerosis and rheumatic heart disease. The use of different animal systems to model cardiovascular disease is important because research on many genetic and chronic diseases of man cannot be carried out in human beings. During the last 15 years, the mouse has become the model organism of choice to study human heart disease for several reasons (e.g. 99\% of the human genes have direct orthologs in the mouse and gene targeting technology in mouse embryonic stem cells allows the design and generation of transgenic mice to model human genetic disorders). To evaluate the clinical potential of the pathways and networks affected in mouse models, parallel studies in larger animals (e.g. dogs or pigs) are crucial. However, it is essential to take into account that there are significant differences between human HF and its animal models.

Numerous genetically engineered mouse models for human cardiomyopathies have been generated. For some of them an analogous mutation has been reported to cause human disease (e.g. MLP, $\alpha$-MHC) whereas for others this has not been shown so far (e.g. ErbB2). Indeed, mice show a different response to drug treatment (e.g. a smaller dynamic range in cardiac output alterations after adrenergic stimulation) and have significantly different myocardial action potential waveforms than larger animals or human beings [1]. In addition, one has to consider that in comparison to mouse inbred strains, due to the polygenic and multifactorial character of human disease, clinical parameters of patients are highly heterogenic. Moreover, it remains unclear whether a specific animal model resembles the human pathophysiological condition with its corresponding chronic features like fibrosis, inflammation, apoptosis and its duration of several years (e.g. the tachycardia-induced canine model develops HF already within a few weeks). Nevertheless, the analysis of animal models allows a better understanding of human disease mechanisms. Until now, animal models for HF were characterized to a limited extent at phenotypic and biochemical levels. Relevant studies included only a reduced number of parameters (e.g. BNP plasma levels). Gao and collaborators tackled this issue with a very innovative and promising approach. They aimed at the characterization of different animal models for HF from a global point of view, i.e. at a tran- scriptional level using a broad gene expression profiling approach. Therefore they compared the gene expression pattern in a canine HF model with those in human and murine $\mathrm{HF}$ to provide insight for the general validity of their findings in mammals.

During HF multiple pathways are responsible for transducing mechanical load and biochemical signals into changes of gene expression. The molecular basis, i.e. the origins and mechanisms of many alterations found in patients suffering from $\mathrm{HF}$ is poorly understood, but genome-wide gene expression analysis approaches are starting to reveal important associations between gene expression signatures and the pathogenesis and outcome of the disease [2-4]. High expectations have been raised that expression profiles might be used as a diagnostic and/or prognostic aid in disease management, e.g. before and after left ventricular assist device placement [5]. Moreover, recent reports based on expression profiling data demonstrate the feasibility of discrimination between different disease entities, e.g. ischemic and non-ischemic cardiomyopathy [6] or disease stages [7].

The report by Gao and collaborators is the first one on the comparison of gene expression profiles of a canine tachycardia pacing-induced HF model and human and murine HF. The authors used the human $20 \mathrm{~K}$ cDNA microarray fabricated by the Microarray Core of the John Hopkins University to measure the gene expression of the left and right ventricles from normal and failing age-matched male canine hearts. The ventricle-specific expression profiles as well as the healthy and disease-specific ones were obtained and compared with those from human HF patients and from two different mouse models for HF. The authors aimed at the identification of global processes associated with HF instead of focusing on individual genes and compared their overlap between different human etiologies and different animal models. Therefore, differential gene expression was detected using the significance analysis of microarrays (SAM; Tusher et al.) and a subsequent expression analysis systematic explorer (EASE) was applied to the data set to identify gene ontology (GO) categories with an overrepresented number of deregulated genes. GO annotation represents a powerful tool to encounter the challenge of long lists of deregulated genes. It provides first hints on the affected pathways or processes and has been successfully applied to numerous data sets during the last few years. Using this approach the authors could correlate several GO categories, e.g. nucleic acid metabolism, transcrip- 
tion and energy pathways with HF in the canine tachycardiainduced model. The majority of deregulated genes was found in the failing left ventricle whereas no significant overrepresentation of genes in distinct GO categories between the ventricles was found. It seems surprising, however, that in contrast to other studies (mainly using human samples), a high percentage $(95 \%)$ of the genes with significant expression differences were found to be down-regulated and no induction of prominent HF markers, e.g. ANP and BNP was detected.

The authors' main interest was to evaluate the similarities and differences of the transcriptional program between the animal models and that in human HF. For obvious reasons a gene-by-gene comparison of lists of deregulated HFassociated genes derived from different species is neither conclusive nor meaningful. In a comparable and exemplary study, Lee et al. [8] used hierarchical clustering and several expression-based prediction methods to identify the best-fit mouse model for hepatocellular carcinomas [8]. Samples of seven different mouse models (68 animals in total) were compared with 91 human samples grouped in a better and a poorer survival group, respectively. Here, a strong correlation was found between Myc, E2f1 and Myc-E2f1 transgenic mice and the patients of the better survival group, whereas Myc-TGFa transgenic mice and diethylnitrosamine-treated animals were most similar to the poorer survival group of patients (Fig. 1). Notably, the authors could validate and substantiate their findings using the human poorer and better survival groups as training sets for supervised expression-based classification: again a strong correlation of Myc-TGFa transgenic mice with the poorer survival group of patients was found. Altogether, unsupervised (clustering) and supervised (expression-based classification) methods allowed the identification of mouse models mimicking best different human cancer disease entities. Such approaches however are restricted to calculated expression similarities and do not directly deliver information about the global biological processes involved.

Gao and co-workers tackled this issue and used EASE to identify GO categories being that they were mainly affected during HF in the different species. Their own generated data from the canine tachycardia pacing-induced model was compared with that of human ischemic and idiopathic etiologies as well as with two different mouse models, the TNFa overexpression model and the MLP knockout model. Human expression data were downloaded from the website of Cardiogenomics (http://www.cardiogenomics.med.harvard.edu) and mouse expression data were obtained from previously reported studies $[9,10]$. Surprisingly, the authors found only little overlap in the GO categories between the different animal models analyzed and human HF. It is noteworthy that out of the $18 \mathrm{GO}$ categories characteristic for the canine HF model, eight of them were identical in the human ischemic HF group. Notably, the GO categories found (e.g. immune and defense response, antigen presentation and chemotaxis) in the specific animal models (e.g. the TNFa transgenic mice) appear to be model- and pathway-specific suggesting that multiple molecular mechanisms may converge on a HF phenotype.

Cross-species hybridization was proven earlier to facilitate the identification of differentially expressed ortholog genes in tissues derived from different species [11]. This approach has therefore become acceptable and very reasonable for species for which no array format is available. Indeed, Gao et al. confirmed the differential expression of several genes detected in cross-species hybridizations (canine targets onto human probes) with RT-PCR demonstrating the validity of their experimental setting. It seems difficult however to estimate the false positive and especially the false negative rate of the approach in comparison to species-matching expression profiling experiments. This issue becomes relevant when comparing the canine tachycardia-induced model array data with the mouse model and human HF array data, since specific binding of the probe and the target on the microarray depends on their sequence similarity. Variations in the sequence conservation of genes belonging to different GO categories (i.e. immune response or the TCA cycle) might in such an experimental setting lead to GO category-specific false negative rates.

Another key issue in this regard is that the array data analyzed here were generated by independent research groups

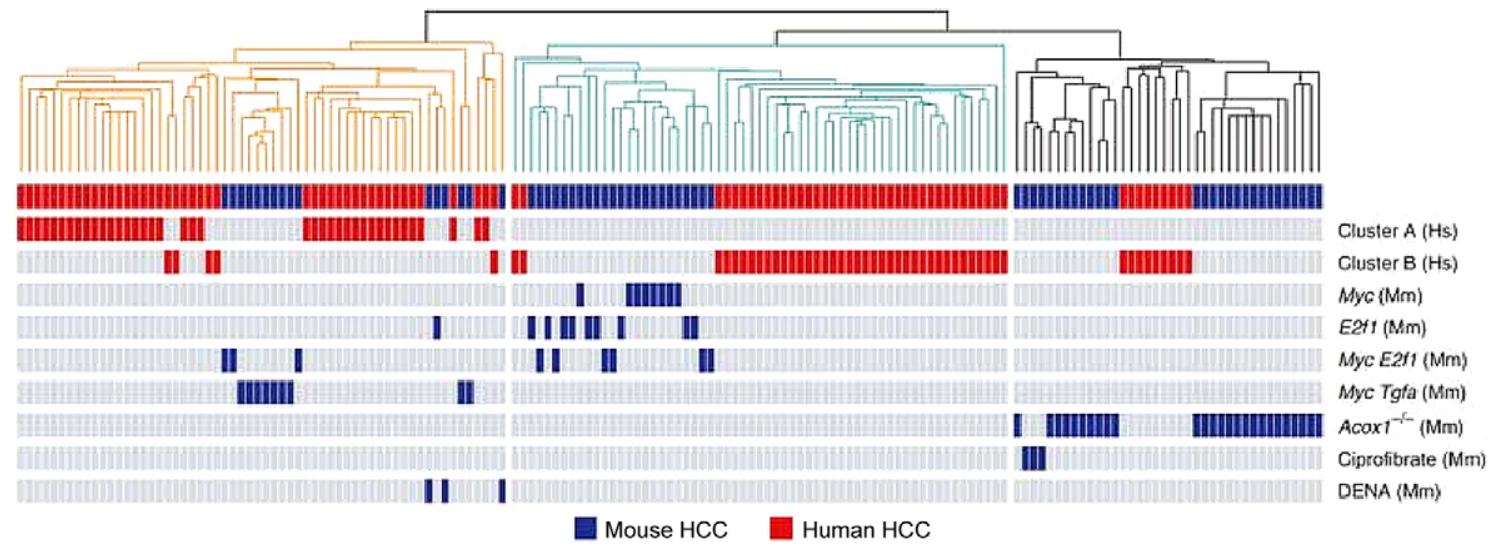

Fig. 1. Dendogram of an unsupervised cluster analysis of integrated 68 mouse and 91 human hepatocellular carcinoma (HCC) samples based on 1650 orthologous genes. Red and blue bars represent human and mouse HCC tissues, human samples are subdivided into a poorer (Cluster A (Hs)) and a better survival group ((Cluster B (Hs)). Adapted from [8]. 


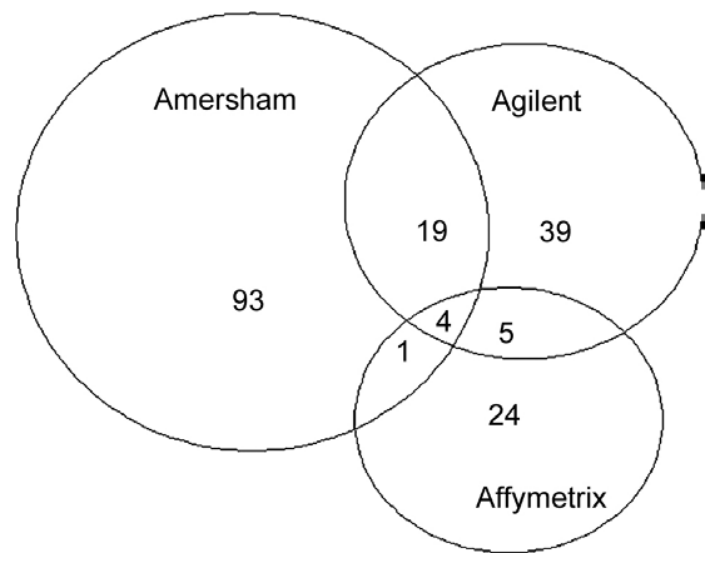

Fig. 2. Venn diagram with the differentially expressed genes detected by gene expression profiling using the same biological material but three different platforms with five replicates for each platform: Amersham (Codelink Uniset Human 1), Agilent (Agilent Human 1 cDNA array) and Affymetrix (U95A v2). Note the small overlap of deregulated genes identified using the different platforms. Adapted from [12].

using different microarray platforms (i.e. Affymetrix and in-house produced arrays). As reported in several studies expression profiling of the same biological material but using different array platforms delivers data sets with alarmingly poor overlap [12] (Fig. 2). This outcome originates mainly from a high false negative rate caused by, e.g. differences in sensitivity and in the targeted sequences. These effects, together with the biological heterogeneity of the samples used, become apparent in several published array studies on HF $[2,3,13,14]$. In these data sets only a limited overlap of deregulated genes can be found and the deregulation of even prominent HF markers belonging to the fetal gene program, e.g. ANP, BNP or alpha skeletal actin cannot be reproducibly detected.

Altogether, Gao et al., conclude that the comparison among the canine tachypacing, mouse transgenic and human HF reveals that human HF involves deregulation of genes in a broad range of biological processes and that chronically developing human HF may impact more biological processes than the experimentally induced genetic of sub-acute HF models. The identification of animal models for human disease in general and HF in particular which may serve as valuable experimental systems for an improved diagnosis and treatment of human HF remains a challenge. It can however certainly be assisted by comparing comprehensive gene expression patterns of different animal models with those of human patients. The study by Gao et al. provides insights into the molecular pathways leading to HF and into global transcriptional differences between animal models for HF and human HF demonstrating that the careful and detailed analysis of global gene expression patterns between different animal models and across species may be instrumental.

\section{References}

[1] Nerbonne JM. Studying cardiac arrhythmias in the mouse-a reasonable model for probing mechanisms? Trends Cardiovasc Med 2004; 14(3):83-93.

[2] Tan FL, Moravec CS, Li J, Apperson-Hansen C, McCarthy PM, Young JB, et al. The gene expression fingerprint of human heart failure. Proc Natl Acad Sci USA 2002;99(17):11387-92.

[3] Barrans JD, Allen PD, Stamatiou D, Dzau VJ, Liew CC. Global gene expression profiling of end-stage dilated cardiomyopathy using a human cardiovascular-based cDNA microarray. Am J Pathol 2002; 160(6):2035-43.

[4] Liew CC. Expressed genome molecular signatures of heart failure. Clin Chem Lab Med 2005;43(5):462-9.

[5] Blaxall BC, Tschannen-Moran BM, Milano CA, Koch WJ. Differential gene expression and genomic patient stratification following left ventricular assist device support. J Am Coll Cardiol 2003;41(7):1096106.

[6] Kittleson MM, Ye SQ, Irizarry RA, Minhas KM, Edness G, Conte JV, et al. Identification of a gene expression profile that differentiates between ischemic and non-ischemic cardiomyopathy. Circulation 2004;110(22):3444-51.

[7] Steenman M, Lamirault G, Le Meur N, Le Cunff M, Escande D, Leger JJ. Distinct molecular portraits of human failing hearts identified by dedicated cDNA microarrays. Eur J Heart Fail 2005;7(2):15765.

[8] Lee JS, Chu IS, Mikaelyan A, Calvisi DF, Heo J, Reddy JK, et al. Application of comparative functional genomics to identify best-fit mouse models to study human cancer. Nat Genet 2004;36(12):130611.

[9] Tang Z, McGowan BS, Huber SA, McTiernan CF, Addya S, Surrey S, et al. Gene expression profiling during the transition to failure in TNF-alpha over-expressing mice demonstrates the development of autoimmune myocarditis. J Mol Cell Cardiol 2004;36(4):515-30.

[10] Blaxall BC, Spang R, Rockman HA, Koch WJ. Differential myocardial gene expression in the development and rescue of murine heart failure. Physiol Genomics 2003;15(2):105-14.

[11] Adjaye J, Herwig R, Herrmann D, Wruck W, Benkahla A, Brink TC, et al. Cross-species hybridisation of human and bovine orthologous genes on high density cDNA microarrays. BMC Genomics 2004;5(1): 83.

[12] Tan PK, Downey TJ, Spitznagel Jr. EL, Xu P, Fu D, Dimitrov DS, et al. Evaluation of gene expression measurements from commercial microarray platforms. Nucleic Acids Res 2003;31(19):5676-84.

[13] Grzeskowiak R, Witt H, Drungowski M, Thermann R, Hennig S, Perrot A, et al. Expression profiling of human idiopathic dilated cardiomyopathy. Cardiovasc Res 2003;59(2):400-11.

[14] Kaab S, Barth AS, Margerie D, Dugas M, Gebauer M, Zwermann L, et al. Global gene expression in human myocardium-oligonucleotide microarray analysis of regional diversity and transcriptional regulation in heart failure. J Mol Med 2004;82(5):308-16.

Patricia Ruiz * Henning Witt

Center for Cardiovascular Research, Charité Universitätsmedizin Berlin, Berlin, Germany E-mail address: ruiz@molgen.mpg.de (P. Ruiz).

Available online 29 November 2005

* Corresponding author. 